PROCEEDINGS OF THE

AMERICAN MATHEMATICAL SOCIETY

Volume 135, Number 8, August 2007, Pages 2347-2354

S 0002-9939(07)08915-0

Article electronically published on March 21, 2007

\title{
THE NOETHER MAP II
}

\author{
MARA D. NEUSEL AND MÜFIT SEZER
}

(Communicated by Bernd Ulrich)

\begin{abstract}
Let $\rho: G \hookrightarrow \operatorname{GL}(n, \mathbb{F})$ be a faithful representation of a finite group $G$. In this paper we proceed with the study of the image of the associated Noether map

$$
\eta_{G}^{G}: \mathbb{F}[V(G)]^{G} \rightarrow \mathbb{F}[V]^{G} .
$$

In our 2005 paper it has been shown that the Noether map is surjective if $V$ is a projective $\mathbb{F} G$-module. This paper deals with the converse. The converse is in general not true: we illustrate this with an example. However, for $p$-groups (where $p$ is the characteristic of the ground field $\mathbb{F}$ ) as well as for permutation representations of any group the surjectivity of the Noether map implies the projectivity of $V$.
\end{abstract}

Let $\rho: G \hookrightarrow \operatorname{GL}(n, \mathbb{F})$ be a faithful representation of a finite group $G$ of order $d$ over a field $\mathbb{F}$. The representation $\rho$ naturally induces an action of $G$ on the vector space $V=\mathbb{F}^{n}$ of dimension $n$ and hence on the ring of polynomial functions $\mathbb{F}[V]=\mathbb{F}\left[x_{1}, \ldots, x_{n}\right]$. Our interest is focused on the subring of invariants

$$
\mathbb{F}[V]^{G}=\left\{f \in \mathbb{F}[V]^{G} \text { such that } g f=f \forall g \in G\right\},
$$

which is a graded connected Noetherian commutative algebra. Denote by $\mathbb{F} G$ the group algebra. Let

$$
V(G)=\mathbb{F} G \otimes_{\mathbb{F}} V
$$

be the induced module. The group $G$ acts on $V(G)$ by left multiplication on the first component. We obtain a $G$-equivariant surjection

$$
V(G) \rightarrow V,(g, v) \mapsto g v .
$$

Let us choose a basis $e_{1}, \ldots, e_{n}$ for $V$. Let $x_{1}, \ldots, x_{n}$ be the standard dual basis for $V^{*}$, and set $G=\left\{g_{1}, \ldots, g_{d}\right\}$. Then $V(G)$ can be written as

$$
V(G)=\operatorname{span}_{\mathbb{F}}\left\{e_{i j} \mid i=1, \ldots, n, j=1, \ldots, d\right\},
$$

and the map $(\star)$ translates into

$$
V(G) \rightarrow V, e_{i j} \mapsto g_{j} e_{i}
$$

Similarly, we have

$$
V(G)^{*}=\operatorname{span}_{\mathbb{F}}\left\{x_{i j} \mid i=1, \ldots, n, j=1, \ldots, d\right\}
$$

Received by the editors April 12, 2006.

2000 Mathematics Subject Classification. Primary 13A50, 20 J06.

Key words and phrases. Invariant theory of finite groups, Noether map, modular invariant theory, projective $\mathbb{F} G$-modules, $p$-groups, permutation representations.

The first author is partially supported by NSA Grant No. H98230-05-1-0026. 
with

$$
V(G)^{*} \rightarrow V^{*}, x_{i j} \mapsto g_{j} x_{i} .
$$

We obtain a surjective $G$-equivariant map between the rings of polynomial functions

$$
\eta_{G}: \mathbb{F}[V(G)] \rightarrow \mathbb{F}[V] .
$$

The group $G$ acts on $\mathbb{F}[V(G)]$ by permuting the basis elements $x_{i j}$. By restriction to the induced ring of invariants, we obtain the classical Noether map, cf. Section 4.2 in $[9$,

$$
\eta_{G}^{G}: \mathbb{F}[V(G)]^{G} \rightarrow \mathbb{F}[V]^{G} .
$$

We note that $V(G)$ is the $n$-fold regular representation of $G$. Thus $\mathbb{F}[V(G)]^{G}$ are the $n$-fold vector invariants of the regular representation of $G$.

In the classical nonmodular case, where $p \nmid d$, the map $\eta_{G}^{G}$ is surjective; see Proposition 4.2.2 in 9]. This has been generalized in the sense that the Noether map is surjective if $V$ is a projective $\mathbb{F} G$-module; see Proposition 3.1 in [8]. The converse may fail as we illustrate with the next example.

Example. Let $\mathrm{GL}\left(2, \mathbb{F}_{3}\right)$ be the general linear group of $2 \times 2$ matrices with entries from the field with three elements. By Corollary 9.14 in 4 the top Dickson class $\mathbf{d}_{2,0}$ is in the image of the transfer. Hence it is in the image of the Noether map. In order to also see that the other Dickson class $\mathbf{d}_{2,1}$ is in the image of the Noether map, we note that $\mathrm{GL}\left(2, \mathbb{F}_{3}\right)$ contains a supgroup $H$ of order 6 generated by

$$
\left[\begin{array}{cc}
1 & 1 \\
0 & 1
\end{array}\right] \text { and }\left[\begin{array}{cc}
\lambda & 0 \\
0 & \lambda
\end{array}\right]
$$

where $\lambda \in \mathbb{F}^{\times}$. Denote these six elements by $h_{1}, \ldots, h_{6}$. Then the stabilizer subgroup of the monomial

$$
\left(h_{1} \otimes x_{1}\right) \cdots\left(h_{6} \otimes x_{1}\right) \in \mathbb{F}\left[V\left(\mathrm{GL}\left(2, \mathbb{F}_{3}\right)\right)\right]
$$

is $H$. Direct computation yields

$$
\eta_{\mathrm{GL}\left(2, \mathbb{F}_{3}\right)}^{\mathrm{GL}\left(\mathbb{F}_{3}\right)}\left(o\left(\left(h_{1} \otimes x_{1}\right) \cdots\left(h_{6} \otimes x_{1}\right)\right)\right)=-\mathbf{d}_{2,1} .
$$

Thus $\eta_{\mathrm{GL}\left(2, \mathbb{F}_{3}\right)}^{\mathrm{GL}\left(2, \mathbb{F}_{3}\right)}$ is surjective, but the tautological representation of $\mathrm{GL}\left(2, \mathbb{F}_{3}\right)$ is not projective.

In the next section we prove that whenever $G$ is a $p$-group or $\rho$ is a permutation representation, the Noether map is surjective if and only if $V$ is a projective $\mathbb{F} G$ module.

Before we proceed we present a general characterization:

Proposition. $V$ is projective if and only if

$$
\eta_{G}^{G}: \mathbb{F}[\operatorname{End}(V)(G)]^{G} \rightarrow \mathbb{F}[\operatorname{End}(V)]^{G}
$$

is surjective.

Proof. $V$ is projective if and only if $\operatorname{End}(V)$ is projective by [2]. Thus the Noether map on that vector space is surjective by Proposition 3.1 in [8]. Conversely, if the above Noether map is surjective, then it is surjective in degree one. Hence the transfer map is surjective in degree one by Corollary 1.2 below. In particular, the identity on $V$ is in the image of the transfer. Thus $V$ is projective by the Higman criterion; see, e.g., Proposition 3.6.4 in [3]. 


\section{1. $p$-Groups AND PERMUtATion REPRESENTATIONS}

In this section we want to show that the converse Proposition 3.1 in $[8$ is true in the case of $p$-groups $P$ and in the case of permutation representations.

Lemma 1.1. Let $P$ be a cyclic $p$-group, and let $\mathbb{F}$ have characteristic $p$. Then

$$
\operatorname{Im}\left(\operatorname{Tr}^{P}\right)_{(1)}=\mathbb{F}[V]_{(1)}^{P}
$$

if and only if $V$ is the $k$-fold regular representation of $P$ for some $k \in \mathrm{N}$.

Proof. Since the transfer is additive it suffices to consider indecomposable modules only.

Let the order of the group be $p^{s}$. Then up to isomorphism there are exactly $p^{s}$ indecomposable $\mathbb{F} P$-modules $V_{1}, \ldots, V_{p^{s}}$ with $\operatorname{dim}_{\mathbb{F}} V_{i}=i$. The action of $P$ on $V_{i}$ is afforded by the matrix consisting of one Jordan block with 1 's on the diagonal and superdiagonal. Note that $V_{i}^{P}=V_{1}$ for all $i$.

Set $\Delta=g-1$ where $g \in P$ is a generator. Then

$$
\Delta\left(V_{i}^{*}\right)= \begin{cases}V_{i-1}^{*} & \text { for } i=2, \ldots, p^{s}, \\ 0 & \text { for } i=1 .\end{cases}
$$

Since, $\operatorname{Tr}^{P}=\Delta^{p^{s}-1}$, we obtain

$$
\operatorname{Tr}^{P}\left(V_{i}^{*}\right)=\Delta^{p^{s}-1}\left(V_{i}^{*}\right)= \begin{cases}0 & \text { for } i=1, \ldots, p^{s}-1 \\ V_{1}^{*} & \text { for } i=p^{s}\end{cases}
$$

as desired.

We obtain the following corollary that we note here for later reference.

Corollary 1.2. Let $\rho: G \hookrightarrow \operatorname{GL}(n, \mathbb{F})$ be a faithful representation of a finite group. Let $i \in \mathbb{F}^{\times}$. Then

$$
\operatorname{Im}\left(\left.\eta_{G}^{G}\right|_{(i)}\right)=\operatorname{Im}\left(\left.\operatorname{Tr}^{G}\right|_{(i)}\right) .
$$

Proof. By construction we obtain a commutative diagram as follows:

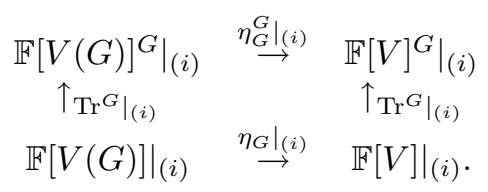

By Theorem 3.2 in [7] and the remark following it the transfer map on the left

$$
\left.\operatorname{Tr}^{G}\right|_{(i)}:\left.\left.\mathbb{F}[V(G)]\right|_{(i)} \rightarrow \mathbb{F}[V(G)]^{G}\right|_{(i)}
$$

is surjective. By construction the lower map $\left.\eta_{G}\right|_{(i)}$ is surjective. Thus the result follows.

Theorem 1.3. Let $\rho: P \hookrightarrow \operatorname{GL}(n, \mathbb{F})$ be a representation of a p-group over a field $\mathbb{F}$ of characteristic $p$. Then the following are equivalent:

(1) The Noether map is surjective.

(2) The Noether map is surjective in degree one.

(3) $V$ is a projective $\mathbb{F} P$-module. 
Proof. The implication $(1) \Rightarrow(2)$ is trivial. The implication $(3) \Rightarrow(1)$ was proven in Proposition 3.1 in $\left[8\right.$. Thus we need to show that $V$ is projective if $\left.\eta_{P}^{P}\right|_{(1)}$ is surjective.

Consider the short exact sequence of $\mathbb{F} P$-modules

$$
0 \rightarrow K^{*} \rightarrow V(P)^{*} \stackrel{\left.\eta_{P}\right|_{(1)}}{\rightarrow} V^{*} \rightarrow 0 .
$$

The module $V(P)$ is free and therefore cohomologically trivial. Thus the long exact cohomology sequence breaks up into

$$
0 \rightarrow\left(K^{*}\right)^{P} \rightarrow\left(V(P)^{*}\right)^{P} \stackrel{\left.\eta_{P}^{P}\right|_{(1)}}{\rightarrow}\left(V^{*}\right)^{P} \rightarrow \mathrm{H}^{1}\left(P, K^{*}\right) \rightarrow 0
$$

and

$$
\mathrm{H}^{i}\left(P, V^{*}\right) \cong \mathrm{H}^{i+1}\left(P, K^{*}\right) \quad \forall i \geq 1 .
$$

Since $\left.\eta_{P}^{P}\right|_{(1)}$ is surjective by assumption, we obtain

$$
\mathrm{H}^{1}\left(P, K^{*}\right)=0 .
$$

Thus $K^{*}$ is a projective $\mathbb{F} P$-module (see, e.g., Proposition 4.4 .11 in [10]). Since $P$ is finite and $K^{*}$ finitely generated, this implies that $K^{*}$ is injective; see Corollary 2.7 in [5. Thus the sequence $(*)$ splits and $V^{*}$ is projective as desired.

We illustrate this result with an example.

Example 1. Let $\mathbb{F}$ be the field with $q$ elements of characteristic $p$. Let $P \leq$ $\mathrm{GL}(n, \mathbb{F})$ be a $p$-Sylow subgroup of the general linear group. We assume without loss of generality that $P$ consists of all upper triangular matrices with 1's on the diagonal. Then

$$
\mathbb{F}[V(P)]_{(1)}^{P}=\operatorname{span}_{\mathbb{F}}\left\{o\left(x_{i 1}\right)=\sum_{j=1}^{|P|} x_{i j} \text { such that } i=1, \ldots, n\right\} .
$$

Thus

$$
\begin{aligned}
& \eta_{P}^{P}\left(o\left(x_{i 1}\right)\right)=\sum_{j=1}^{|P|} g_{j} x_{i}=\sum_{\left(a_{i+1}, \ldots, a_{n}\right) \in \mathbb{F}^{n-i}}\left(x_{i}+a_{i+1} x_{i+1}+\cdots+a_{n} x_{n}\right) \\
& =q^{\frac{n(n-1)}{2}-(n-i)}\left(q^{n-i} x_{i}+q^{n-i-1}\left(\sum_{a_{i+1} \in \mathbb{F}} a_{i+1} x_{i+1}+\cdots+\sum_{a_{n} \in \mathbb{F}} a_{n} x_{n}\right)\right) \\
& =q^{\frac{n(n-1)}{2}} x_{i}+q^{\frac{n(n-1)}{2}-1}\left(\sum_{a_{i+1} \in \mathbb{F}} a_{i+1} x_{i+1}+\cdots+\sum_{a_{n} \in \mathbb{F}} a_{n} x_{n}\right) .
\end{aligned}
$$

If $n \leq 1$, then $P$ is the trivial group. Therefore $V$ is $\mathbb{F} P$-projective and the Noether map is surjective.

If $n \geq 2$, then the factor $q^{\frac{n(n-1)}{2}}$ vanishes. The factor $q^{\frac{n(n-1)}{2}-1}$ is nonzero if and only if $n=2$. Thus we proceed by having a closer look at the two-dimensional 
case: We have by the above calculations

$$
\begin{aligned}
& \eta_{P}^{P}\left(o\left(x_{11}\right)\right)=\sum_{j=1}^{|P|} g_{j} x_{1}=\sum_{a_{2} \in \mathbb{F}}\left(x_{1}+a_{2} x_{2}\right)=\left(\sum_{a_{2} \in \mathbb{F}} a_{2}\right) x_{2}, \\
& \eta_{P}^{P}\left(o\left(x_{21}\right)\right)=\sum_{j=1}^{|P|} g_{j} x_{2}=0 .
\end{aligned}
$$

If $p$ is odd, then for every nonzero $a_{2} \in \mathbb{F}$ there exists a negative $-a_{2} \neq a_{2}$. Therefore

If $p=2$, then

$$
\sum_{a_{2} \in \mathbb{F}} a_{2}=0
$$

$$
\left(\sum_{a_{2} \in \mathbb{F}} a_{2}\right) x_{2}= \begin{cases}x_{2} & \text { if } q=2, \\ 0 & \text { if } q>2 .\end{cases}
$$

Thus we have that the Noether map is surjective if and only if $n=2=p=q$. Explicitely we find

$$
\eta_{P}^{P}\left(o\left(x_{11}\right)\right)=x_{2} \quad \text { and } \quad \eta_{P}^{P}\left(o\left(x_{11} x_{12}\right)\right)=x_{1}^{2}+x_{1} x_{2} .
$$

Note that in this case

$$
\operatorname{Syl}_{2}\left(\mathrm{GL}\left(2, \mathbb{F}_{2}\right)\right) \cong \mathbb{Z} / 2
$$

and our representation is projective.

Before proceeding to permutation representations, we want to mention two corollaries.

Corollary 1.4. Let $\rho: G \hookrightarrow \mathrm{GL}(n, \mathbb{F})$ be a faithful representation of a finite group. Assume that the rings of invariants of $G$ and its p-Sylow subgroup coincide in degree one. Then the Noether map is surjective if and only if $V$ is $\mathbb{F} G$-projective.

Proof. Denote by $P$ the $p$-Sylow subgroup of $G$. We consider the relative Noether map given by the following commutative diagram:

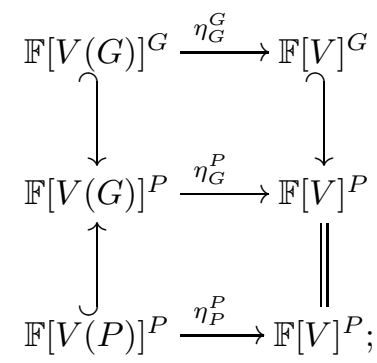

cf. [8. If $\eta_{G}^{G}$ is surjective, then it is surjective in degree one. Hence $\eta_{G}^{P}$ is surjective in degree one by assumption. Therefore $\eta_{P}^{P}$ is surjective in degree one by Proposition 2.1 in 8 . Thus $V$ is projective by Theorem [1.3. The converse was shown in Proposition 3.1 in 8 .

Corollary 1.5. Let $G=H \times P$ be a direct product of a p-group $P$ and a $p^{\prime}$-group $H$. Assume that $P$ is a cyclic p-group. Consider a faithful representation $\rho$ of $G$ over a field $\mathbb{F}$ of characteristic $p$ such that $V$ is indecomposable as an $\mathbb{F} P$-module. Then the Noether map is surjective if and only if $V$ is $\mathbb{F} G$-projective. 
Proof. If $V$ is $\mathbb{F} G$-projective then the Noether map $\eta_{G}^{G}$ is surjective by Proposition 3.1 in 8 .

To prove the converse, let $\eta_{G}^{G}$ be surjective. By Proposition 2.1 in $[\underline{8}$ it is enough to show that the relative Noether map $\eta_{G}^{P}$ is surjective. We proceed by contradiction and assume that $\eta_{G}^{P}$ is not surjective. Then, by Proposition 2.1 in [8] the map $\eta_{P}^{P}$ is not surjective. Hence $V$ is not a projective $\mathbb{F} P$-module by Theorem 1.3 .

Let $\sigma$ be a generator for $P$. The isomorphism type of a $P$-module is determined by the Jordan canonical form of $\sigma$. Up to isomorphism there are $|P|$ indecomposable $P$ modules $V_{1}, V_{2}, \ldots V_{|P|}$, where $\operatorname{dim} V_{i}=i$ and $\sigma$ acts on $V_{i}$ by an $i \times i$ matrix consisting of a single Jordan block with ones on the diagonal and superdiagonal. Moreover $V_{|P|}$ is the only indecomposable module which is projective. Thus by assumption we have that $V=V_{n}$ for $1 \leq n<|P|$.

Let $x_{1}, x_{2}, \ldots, x_{n}$ be the basis of $V$ such that

$$
\sigma x_{i}= \begin{cases}x_{1} & \text { if } i=1 \\ x_{i-1}+x_{i} & \text { otherwise. }\end{cases}
$$

Since the action of $P$ commutes with the action of $H$ and the action of $H$ is nonmodular, it follows that $V=V_{n}$ is a direct sum of copies of isomorphic eigenspaces for $H$, and the variables $x_{1}, x_{2}, \ldots, x_{n}$ may be taken as eigenvectors. Let $\mathbf{N}=\prod_{g \in P} g\left(x_{n}\right)$ be the norm of $x_{n}$. Since $p$ and $|H|$ are relatively prime, there exists a positive integer $m$ such that $m|P| \equiv-1 \bmod |H|$. Consider the polynomial $x_{1} \mathrm{~N}^{m}$. This polynomial is $P$-invariant since both $x_{1}$ and $\mathrm{N}$ are. Let $h \in H$. Then

$$
h\left(x_{1} \mathrm{~N}^{m}\right)=\lambda_{h} x_{1} \lambda_{h}^{m|P|} \mathrm{N}^{m}=x_{1} \mathrm{~N}^{m} .
$$

It follows that $x_{1} \mathrm{~N}^{m}$ is $G$-invariant.

Next we want to see that $x_{1} \mathrm{~N}^{m}$ is not in the image of $\operatorname{Tr}^{P}$. Since $V$ is not projective, the fixed point $x_{1}$ is not in the image of $\operatorname{Tr}^{P}$. The degree-one-component $\mathbb{F}[V]_{(1)}$ is a direct summand in $\mathbb{F}[V]_{m|P|+1}$ by multiplication by $\mathbf{N},[6]$. Thus the invariant $x_{1} \mathrm{~N}^{m}$ is not in the image of $\operatorname{Tr}^{P}$ either. However, if a $G$-invariant polynomial is not in the image of $\operatorname{Tr}^{P}$, then it is not in the image of $\operatorname{Tr}^{G}$.

Since the degree of the polynomial $x_{1} \mathrm{~N}^{m}$ is relatively prime to $p$, we have that it is not in the image of $\eta_{G}^{G}$ by Corollary 1.2, This is a contradiction.

Corollary 1.6. Let $P \cong \mathbb{Z} / p$ and let $V$ be an indecomposable $\mathbb{F} P$-module. Then the Noether map $\eta_{P}^{P}$ is surjective in degrees divisible by $p$.

Proof. As above denote by $V=V_{n}$ the indecomposable $\mathbb{F} \mathbb{Z} / p$-modules and $x_{1}, x_{2}$, $\ldots, x_{n}$ be the basis for $V$ on which $\mathbb{Z} / p$ acts through a single Jordan block of dimension $n$. We note that

$$
\mathbb{F}[V]=B \oplus \mathbb{N F}[V]
$$

as $\mathbb{F} P$-modules, where $B$ consists of the polynomials of $x_{n}$-degree less than $p$, [6]

We proceed by induction on the degree. The decomposition

$$
\mathbb{F}[V]_{(p)}^{P}=B_{(p)}^{P} \oplus \mathrm{N} \mathbb{F}[V]^{P}
$$

yields that any invariant in degree $p$ is a direct summand of a fixed point of a free module and the polynomial N. Since fixed points of free modules and $\mathrm{N}$ are in the image of $\eta_{P}^{P}$, the result follows for degree $p$.

Using the decomposition for degree $k p$ we have that

$$
\mathbb{F}[V]_{(k p)}^{P}=B_{(k p)}^{P} \oplus \mathbb{N F}[V]_{((k-1) p)}^{P} .
$$


Since $\eta_{P}^{P}$ is an algebra map, and $\mathbb{F}[V]_{((k-1) p)}^{P}$ is in the image of $\eta_{P}^{P}$ by induction, the result follows.

We turn to permutation representations.

Theorem 1.7. Let $\rho: G \hookrightarrow \mathrm{GL}(n, \mathbb{F})$ be a permutation representation of a finite group of order $d$. Then the Noether map $\eta_{G}^{G}$ is surjective if and only if $V=\mathbb{F}^{n}$ is projective.

Proof. By Proposition 3.1 in $\left[8\right.$ ] we know that $\eta_{G}^{G}$ is surjective if $V$ is projective as an $\mathbb{F} G$-module.

We show that the converse is also true as follows:

Let $\eta_{G}^{G}$ be surjective. Then its restriction to degree one, $\eta_{G}^{G}$ such that (1), is also surjective:

$$
\eta_{G}^{G} \text { such that }{ }_{(1)}:\left(V(G)^{*}\right)^{G} \rightarrow\left(V^{*}\right)^{G} .
$$

We note that $\left(V(G)^{*}\right)^{G}$ has an $\mathbb{F}$-basis consisting of

$$
o\left(x_{i j}\right)=\sum_{j=1}^{d} x_{i j} \quad \text { for } i=1, \ldots, n .
$$

Therefore, the image under the Noether map is spanned by

$$
\eta_{G}^{G}\left(\sum_{j=1}^{d} x_{i j}\right)=k_{i} o\left(x_{i}\right)=\left|\operatorname{Stab}_{G}\left(x_{i}\right)\right| \operatorname{Tr}^{G}\left(x_{i}\right) \quad \text { for } i=1, \ldots, n,
$$

where

$$
k_{i}=\left|\operatorname{Stab}_{G}\left(x_{i}\right)\right|
$$

is the order of the stabilizer of $x_{i}$ in $G$. Since $\rho$ is a permutation representation, $\left(V^{*}\right)^{G}$ is spanned by the orbit sums of $x_{1}, \ldots, x_{n}$. It follows that $k_{i}$ 's are not zero, since the Noether map is surjective. Hence

$$
\left|\operatorname{Stab}_{G}\left(x_{i}\right)\right| \not \equiv 0 \bmod p
$$

In other words, no element in a $p$-Sylow subgroup $P$ of $G$ fixes $x_{i}, i=1, \ldots, n$. Therefore

$$
o^{P}\left(x_{i}\right)=\operatorname{Tr}^{P}\left(x_{i}\right)=\eta_{P}^{P} \text { such that }{ }_{(1)}\left(x_{i 1}\right),
$$

where $o^{P}(-)$ denotes the orbit sum under the action of $P$, and $g_{1}$ is the identity element. Since $\left(V^{*}\right)^{P}$ is also spanned by the orbit sums of the $x_{i}$ 's, we found in ( that $\eta_{P}^{P}$ such that ${ }_{(1)}$ is surjective. Therefore, $\eta_{P}^{P}$ is surjective by Proposition 1.3. Hence $V^{*}$ is a projective $\mathbb{F} P$-module, by the same Propositon 1.3 . Since $P$ is a $p$-Sylow subgroup of $G$, the module $V^{*}$ is projective as a $\mathbb{F} G$-module; see Corollary 3 on Page 66 of [1].

\section{ACKNOWLEDGMENT}

The first author wants to thank Markus Linckelmann for an interesting discussion. 


\section{REFERENCES}

[1] J. L. Alperin, Local Representation Theory, Cambridge Studies in Advanced Mathematics 11, Cambridge University Press, Cambridge 1986. MR860771 (87i:20002)

[2] Maurice Auslander and Jon F. Carlson, Almost-split Sequences and Group Rings, Journal of Algebra 103 (1986), 122-140. MR860693 (88a:16054)

[3] David J. Benson, Representations and Cohomology, Volume I, Cambridge Studies in Advanced Mathematics 30, Cambridge University Press, Cambridge 1991.

[4] Eddy Campbell, Ian P. Hughes, R. James Shank, and David L. Wehlau, Bases for Rings of Coinvariants, Transformation Groups 1 (1996) 307-336. MR1424447 (98a:13011)

[5] Jon F. Carlson, Modules and Group Algebras, Lectures in Mathematics ETH Zürich, Birkhäuser Verlag, Basel-Boston-Berlin 1996. MR1393196 (97c:20013)

[6] Ian Hughes and Gregor Kemper, Symmetric Power of Modular Representations, Hilbert Series and Degree Bounds, Communications in Algebra 28 (2000), 2059-2089. MR 1747371 (2001b:13009)

[7] Mara D. Neusel, The Transfer in the Invariant Theory of Modular Permutation Representations, Pacific J. of Math. 199 (2001) 121-136. MR1847151 (2002g:13012)

[8] Mara D. Neusel and Müfit Sezer, The Noether Map I, preprint Lubbock-Istanbul 2005.

[9] Mara D. Neusel and Larry Smith, Invariant Theory of Finite Groups, Mathematical Surveys and Monographs Vol.94, AMS, Providence RI 2002. MR1869812 (2002k:13012)

[10] Charles A. Weibel, An Introduction to Homological Algebra, Cambridge Studies in Advanced Mathematics 38, Cambridge University Press, Cambridge 1994. MR.1269324 (95f:18001)

Department of Mathematics, Texas Tech University, Lubbock, Texas 79409

E-mail address: mara.d.neusel@ttu.edu

Department of Mathematics and Statistics, BoĞazici Üniversitesi, MS 1042, Bebek, ISTANBUL, TURKEY

E-mail address: mufit.sezer@boun.edu.tr 